\title{
Compósitos de ferrita de Ni-Zn com policloropreno para utilização como materiais absorvedores de radar para a banda $S$
}

\author{
(S-band radar absorbing materials based on $\mathrm{Ni}-\mathrm{Zn}$ \\ ferrite and polycloroprene composites)
}

\author{
E. G. Paulo ${ }^{1,2}$, M. S. Pinho ${ }^{1,3}$, R. da C. Lima ${ }^{1,2}$, M. L. Gregori ${ }^{1}$, T. Ogasawara ${ }^{2}$ \\ ${ }^{1}$ Instituto de Pesquisas da Marinha, Rua Ipiru Praia da Bica, Ilha do Governador, \\ Rio de Janeiro, RJ 21931-090 \\ ${ }^{2}$ Departamento de Metalurgia e de Materiais da COPPE, Universidade Federal do Rio de Janeiro, \\ Centro de Tecnologia, Bloco F, Rio de Janeiro, RJ 21945-970 \\ ${ }^{3}$ Instituto de Macromoléculas, Universidade Federal do Rio de Janeiro, Centro de Tecnologia, Bloco J, \\ C. P. 68525, Rio de Janeiro, RJ \\ 153@ipqm.mar.mil.br
}

Resumo

O objetivo deste trabalho foi a obtenção de Materiais Absorvedores de Radar (RAMs) para a faixa de freqüência de 2,6 a 4,0 GHz (banda S). A carga magnética adicionada na proporção de $80 \%$ em peso à matriz de policloropreno consistiu de uma ferrita de Ni$\mathrm{Zn}$. O compósito, com espessura de $6 \mathrm{~mm}$, apresentou um bom desempenho como RAM para toda a faixa de freqüência analisada com absorção de microondas superior a 96,9\% (-15 dB) e com absorção máxima de 99,5\% (-25 dB) em 3,2 GHz, viabilizando sua aplicação como revestimento externo em plataformas militares, segundo o ensaio de névoa salina.

Palavras-chave: ferrita Ni-Zn, banda S, RAM, ensaio de névoa salina.

\section{Abstract}

The aim of this work was to obtain Radar Absorbing Materials (RAMs) for the frequency range from 2.6 to $4.0 \mathrm{GHz}$ (S-band). The $80 \mathrm{wt} \%$ magnetic filler addition to a polychloroprene matrix was a Ni-Zn ferrite. The composite with 6 mm thickness showed a good RAM performance for the whole range of frequency analyzed, with a microwave absorption greater than $96.9 \%$ (-15 $d B)$, emphasizing the greatest absorption of $99.5 \%(-25 \mathrm{~dB})$ at $3.2 \mathrm{GHz}$, allowing its use as an external coating for naval applications, in accordance with the salt spray testing.

Keywords: Ni-Zn ferrite, S-band, RAM, salt spray testing.

\section{INTRODUÇÃO}

A invisibilidade de plataformas militares ao radar, através do emprego de materiais absorvedores de microondas (RAMs, "Radar Absorbing Materials") é de suma importância para a redução da Seção Reta Radar (RCS, "Radar Cross Section”) [1-3].

Um RAM deve ser constituído por compostos com elevada perda de energia, que absorvam a radiação incidente em freqüências sintonizadas e dissipem a energia absorvida sob a forma de calor, inibindo a energia necessária para o sinal de eco de detecção por radar [3,4]. Os RAMs são recobrimentos cujas propriedades elétricas e magnéticas podem ser alteradas de forma a permitir a absorção de microondas em freqüências específicas ou em um espectro de freqüência mais amplo. Um RAM ideal deve apresentar como características principais a durabilidade, baixa densidade, baixo custo, a abrangência de uma ampla faixa de freqüência e ser de fácil aplicação [3].

Dentre as matrizes poliméricas empregadas para a confecção dos RAMs, os elastômeros apresentam como vantagens a elevada flexibilidade e resistência a intempéries. O policloropreno (CR) é um elastômero que por apresentar elevada resistência à atmosfera salina, torna-se adequado para inúmeras aplicações navais [3].

As ferritas são materiais cerâmicos com propriedades magnéticas, que devido às possíveis combinações de composições químicas e estruturais, podem ser utilizadas em várias aplicações específicas, tais como RAMs. Para cada tipo de estrutura cristalina (espinélio, granada e hexagonal) o ajuste das propriedades magnéticas possibilita a absorção de microondas em diferentes freqüências [5-11]. 
As ferritas duras como as hexaferritas de bário dopadas com cobalto e titânio (Co-TiBaHF) incorporadas em matrizes poliméricas têm sido utilizadas como RAMs para a faixa de freqüência de 8 a $16 \mathrm{GHz}$ (banda $\mathrm{Ku}$ ) [3-6, 9].

As ferritas macias como as de níquel e zinco (Ni-Zn) são compostos do tipo espinélio, com estrutura cúbica, isomórficos ao mineral $\mathrm{MgAl}_{2} \mathrm{O}_{4}$, que exibem uma magnetização permanente, denominada de ferrimagnetismo [2, 7, 12]. Estes produtos foram aditivados ao $\mathrm{CR}$, resultando na obtenção de RAMs para a faixa de freqüência de 2,6 a 4,0 GHz [3].

O objetivo deste trabalho consistiu na obtenção de um Material Absorvedor de Microondas (RAM), que apresentasse uma absorção de microondas superior a $95 \%$ na faixa de freqüência de 2,6 a 4,0 GHz, para ser utilizado como revestimento externo em partes estratégicas de plataformas militares.

\section{MATERIAIS E MÉTODOS}

\section{Produtos Químicos}

A composição estequiométrica da ferrita de Ni-Zn obtida pelo processo de mistura/moagem correspondeu a de $\mathrm{Ni}_{0,32} \mathrm{Zn}_{0,64} \mathrm{Mn}_{0,04} \mathrm{Fe}_{2,00} \mathrm{O}_{4,00}$. Os demais reagentes empregados, como os aditivos de vulcanização e o policloropreno (Neoprene $\mathrm{W}$ da Du Pont, densidade $1,21 \mathrm{~g} / \mathrm{cm}^{3}$, peso molecular ponderado médio $\left(\mathrm{M}_{\mathrm{w}}\right)$ 440.000), foram utilizados como adquiridos [3].

\section{Obtenção dos Compósitos com o Policloropreno}

As ferritas foram misturadas com o policloropreno, com adição dos agentes de vulcanização, resultando na composição percentual em peso 80:20 da ferrita de Ni-Zn:CR (\% em peso) $[3,6]$.

As misturas físicas (blendas) foram realizadas em um misturador de cilindros Berstorff à temperatura ambiente e com velocidades de 22 e $25 \mathrm{rpm}$ (anterior e posterior), de acordo com os procedimentos clássicos empregados pela

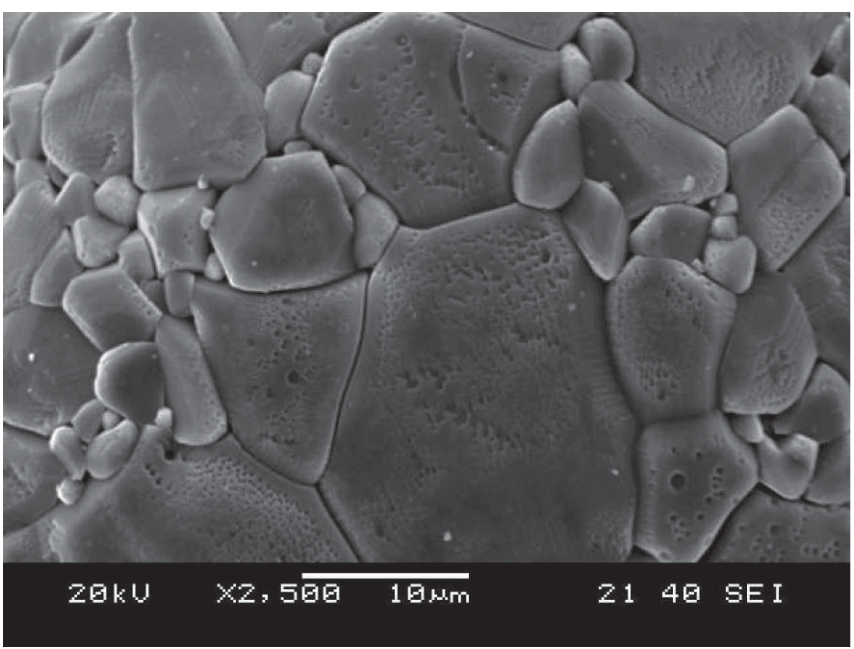

Figura 1: Imagem obtida por microscopia eletrônica de varredura das partículas de ferrita de Ni-Zn, com o aumento de 2.500 vezes.

[Figure 1: SEM picture of Ni-Zn ferrite particles, magnification of 2,500.] indústria de borracha. As blendas foram moldadas por compressão a $150{ }^{\circ} \mathrm{C}$ e $6,7 \mathrm{MPa}$. Os corpos de prova foram obtidos sob a forma de tapetes vulcanizados, com dimensões de $8,0 \times 4,0 \mathrm{~cm}$ e espessuras variadas $[2,3,6]$.

Análise Morfológica por meio de Microscopia Eletrônica de Varredura (SEM)

A observação micromorfológica das amostras, sob a forma de pós, bem como a dispersão em CR foram realizadas pelo emprego de microscópios eletrônicos de varredura LEICA S440 e JEOL JSM-5300, ambos utilizando o detetor de elétrons secundários.

\section{Determinação do Diâmetro Médio de Partículas (Dv 0,5)}

O diâmetro que representa a mediana (Dv 0,5), foi determinado no Analisador de Tamanhos de Partículas por Difração a Laser da Mastersizer Microplus (MAF 5001) da Malvern Instruments [3].

\section{Método de Transmissão/Reflexão (T/R)}

A permeabilidade e permissividade complexas $\left(\mu_{r}^{*}\right.$ e $\varepsilon_{r}^{*}$, respectivamente) foram determinadas, a partir de dados de espalhamento, empregando-se o analisador vetorial de redes HP 8510, que determina as perdas de inserção e retorno em magnitude e fase de amostras submetidas a teste (SUT, Sample Under Test), pela comparação entre o sinal transmitido pela SUT e o refletido na sua entrada $[3,7,13]$. O guia de ondas utilizado apresentou dimensões de 7,5 x 3,5 cm.

\section{Ensaio de Resistência à Névoa Salina}

O teste realizado para a avaliação da resistência a intempéries consistiu do Ensaio de Exposição à Câmara de

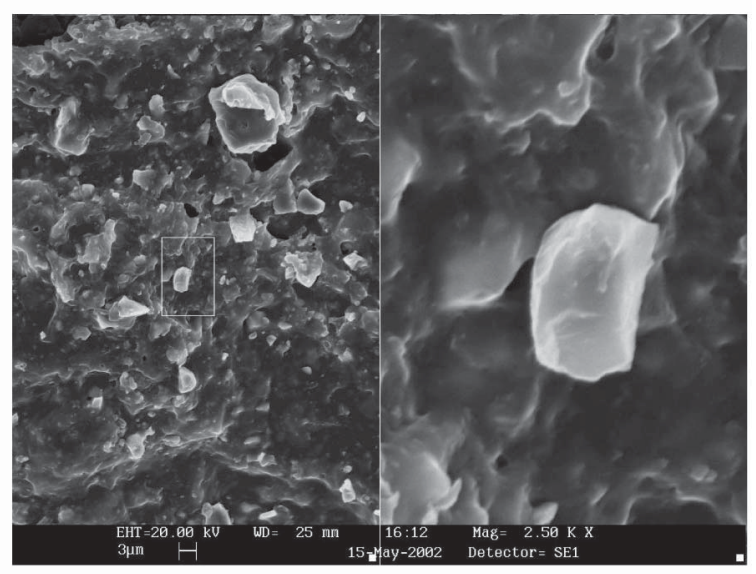
(a) $2.500 \mathrm{X}$
(b) $17.500 \mathrm{X}$

Figura 2: Imagens de microscopia eletrônica de varredura obtidas pela fratura crioscópica do compósito de Ni-Zn:CR (80:20, \% em peso), com os respectivos aumentos.

[Figure 2: SEM pictures of cryofractured samples of Ni-Zn:CR (80:20, wt. \%), with corresponding magnifications.] 
Névoa Salina Druckwal, modelo CA-680. Este ensaio foi realizado segundo a norma ASTM B-117-90, com o intuito de avaliar a resistência de materiais à atmosfera salina de cloreto de sódio $(\mathrm{NaCl})$. $\mathrm{O}$ tempo deste ensaio foi de aproximadamente $744 \mathrm{~h}$, equivalente a $31 \mathrm{~d}$ [14].
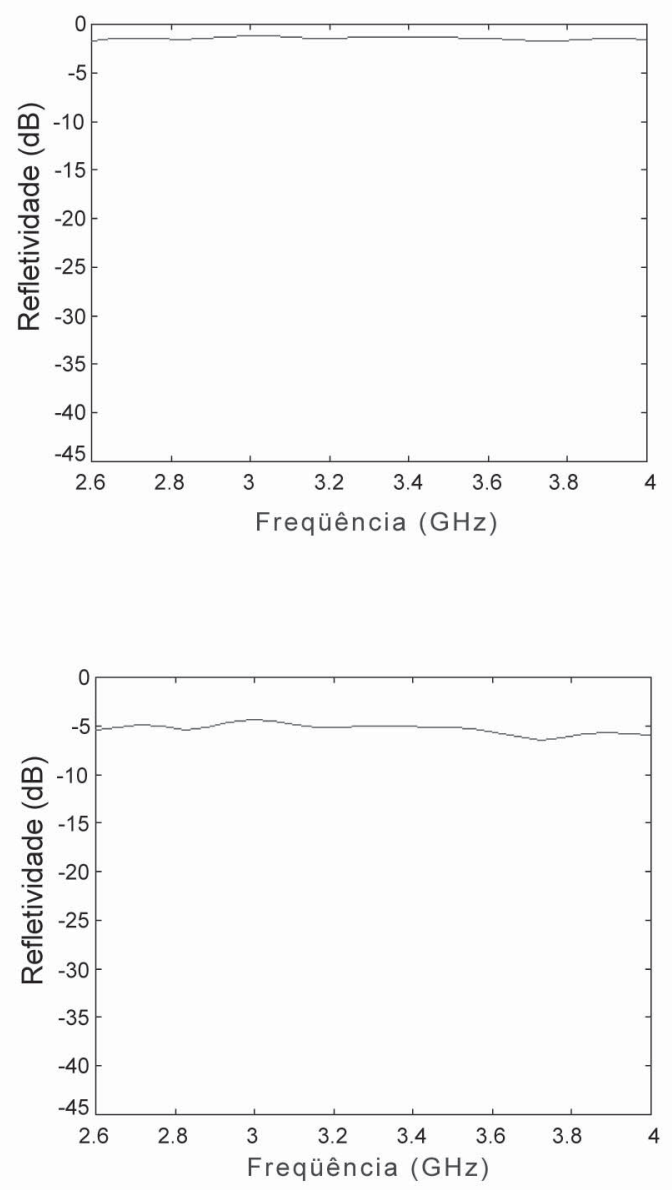

(c) $3 \mathrm{~mm}$

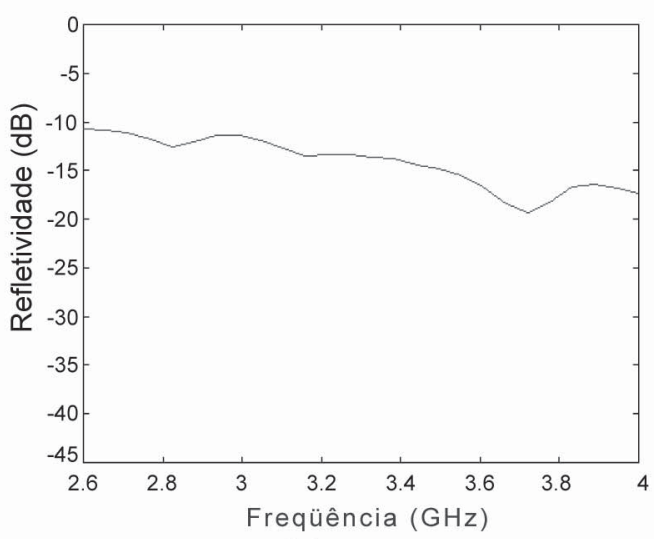

(e) $5 \mathrm{~mm}$
Após exposição dos materiais à câmara de névoa salina, os corpos de prova foram lavados com água destilada, para remover o excesso de cloreto de sódio remanescente, com posterior secagem em estufa a $80{ }^{\circ} \mathrm{C}$ por $48 \mathrm{~h}$.
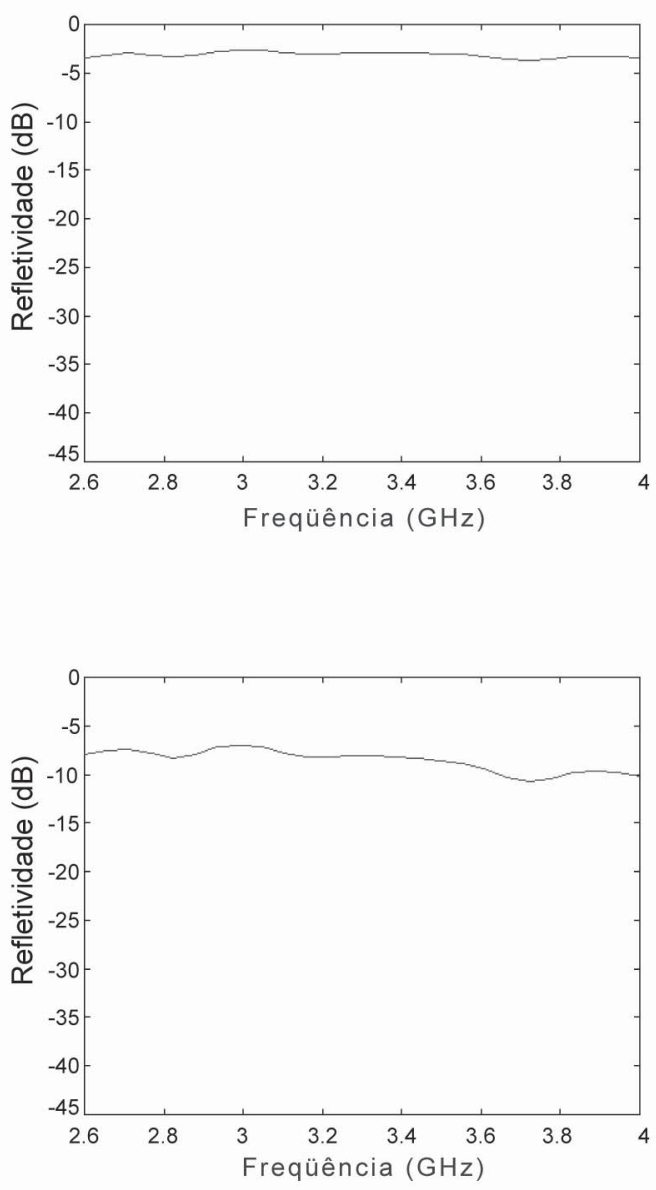

(d) $4 \mathrm{~mm}$

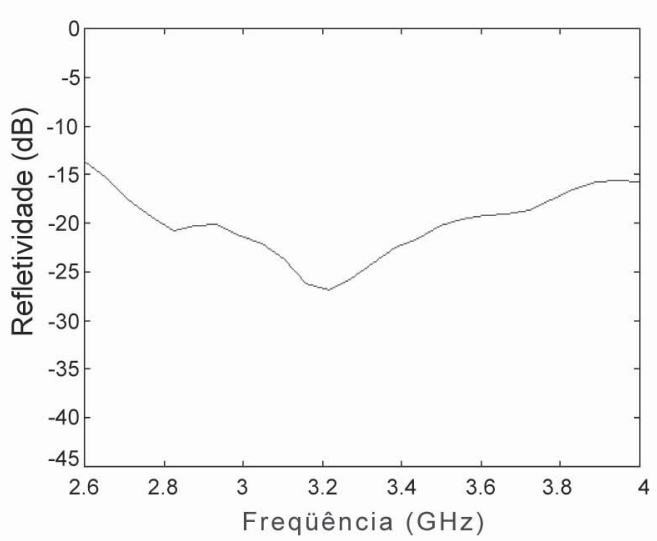

(f) $6 \mathrm{~mm}$

Figura 3: Influência da espessura nas medidas de refletividade $(\mathrm{dB})$ para os compósitos de Ni-Zn:CR (80:20, \% em peso).

[Figure 3: Thickness effect on the reflectivity measurements (dB) for Ni-Zn:CR composites (80:20, wt. \%).] 


\section{RESULTADOS E DISCUSSÃO}

Pela análise da Fig. 1 pode ser observada uma heterogeneidade de tamanhos de partículas, comum em processos físicos de mistura/moagem, largamente empregados pela indústria de cerâmicas magnéticas $[3,11]$.

Pelas imagens obtidas pode ser observada a morfologia cúbica para as partículas da ferrita, que apresentam grande tendência à aglomeração. Esta tendência pode justificar o elevado valor obtido de 83,46 $\mu \mathrm{m}$ para Dv 0,5.

A Fig. 2 apresenta as imagens de microscopia eletrônica de varredura, obtidas pela fratura crioscópica, ilustrando a dispersão das partículas da ferrita de Ni-Zn na matriz de CR.

Conforme a Fig. 2, pode ser observada uma boa dispersão destas partículas no CR, ilustrada pela individualização das mesmas, apesar do elevado carregamento empregado $(80 \% \mathrm{em}$ peso) e da grande tendência do pó à formação de aglomerados. Tal comportamento pode ser atribuído à adsorção do polímero na superfície das partículas sólidas, que propicia uma maior dispersão das partículas magnéticas, devido principalmente à repulsão por impedimento estérico, além da presença de forças de cisalhamento no misturador de cilindros [15].

A Fig. 3 ilustra o efeito da espessura nas medidas de refletividade para os compósitos de Ni-Zn:CR (80:20).

Pelos resultados apresentados na Fig. 3, pode ser observado um aumento da absorção de microondas com o aumento gradativo da espessura. O emprego das espessuras de 5 e 6 $\mathrm{mm}$ resultou no melhor desempenho destes compósitos como RAMs. Na Fig. 3 f pode ser observada uma absorção máxima de 99,5\% (-25 dB) em aproximadamente 3,2 GHz.

Este compósito apresentou uma absorção superior a 96,9\% (-15 dB) para toda a faixa de freqüência analisada.

A resistência do compósito de Ni-Zn:CR (80:20) à atmosfera marítima foi avaliada pelas medidas de refletividade após exposição ao ensaio de névoa salina, conforme ilustra a Fig. 4.

De acordo com os resultados obtidos, não foi observada mudança significativa nas curvas de refletividade após exposição a este ensaio, indicando o emprego deste compósito como um eficiente RAM em plataformas militares para a faixa de freqüência analisada.

\section{CONCLUSÕES}

O compósito 80:20 de Ni-Zn:CR com espessura de $6 \mathrm{~mm}$ apresentou o melhor desempenho como RAM com absorção superior a $96,9 \%(-15 \mathrm{~dB})$, para a faixa de 2,6 a 4,0 GHz (banda $\mathrm{S})$, além de oferecer boa resistência à atmosfera salina, sugerindo seu emprego como um eficiente RAM em partes estratégicas de plataformas militares.

\section{AGRADECIMENTOS}

Os autores agradecem ao Centro de Tecnologia Mineral (CETEM) pelas análises de MEV e ao Centro de Pesquisas de Energia Elétrica (CEPEL) pelos ensaios de névoa salina.

\section{REFERÊNCIAS}

[1] R. N. Johnson, RF Products Technical Bull. (2000) 1-2.

[2] W. Grünberger, B. Springmann, M. Brusberg, M. Schmidt, R. Jahnke, "Rubber bonded ferrite layer as a microwave resonant absorber in a frequency range from 3 up to 16 GHz", J. Magn. Magn. Mater. 101 (1991) 173 -174.

[3] M. S. Pinho, "Materiais absorvedores de radiação eletromagnética em matrizes de policloropreno", Tese de Doutorado, IMA-UFRJ, Rio de Janeiro, R J (2002) 550 p.

[4] H. Zhang, L. Zhichao, Y. Xi, "Complex permittivitty, permeability and microwave absorption of $\mathrm{Zn}$ - and Ti-

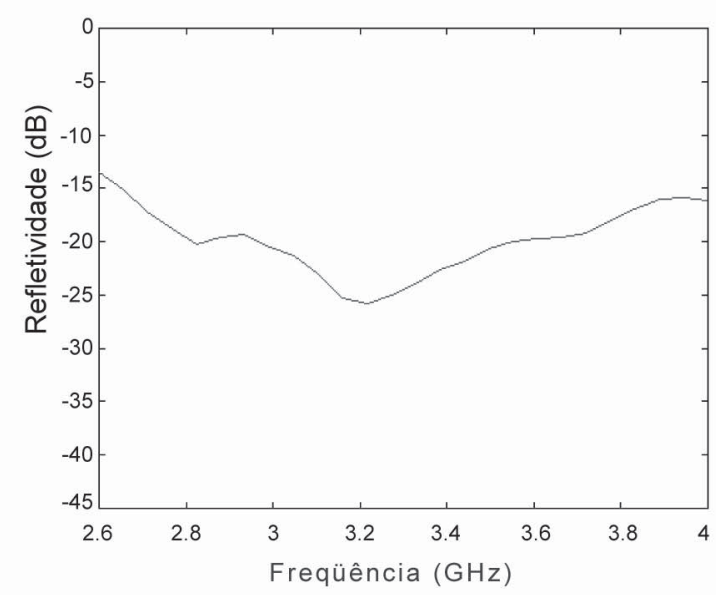

(b) Após Exposição

Figura 4: Curvas de refletividade $(\mathrm{dB})$ em função da freqüência $(\mathrm{GHz})$ para o compósito de Ni-Zn:CR (80:20, \% em peso) com espessura de 6 mm (a) antes e (b) após exposição.

[Figure 4: Reflectivity (dB) as a function of frequency (GHz) for Ni-Zn:CR composite (80:20, wt. \%), (a) before and (b) after exposition.] 
substituted barium ferrite by citrate sol-gel process", Mater. Sci. Eng. B 96 (2002) 289-295.

[5] Y. Li, R. Liu, Z. Zhang, C. Xiong, "Synthesis and characterization of nanocrystalline $\mathrm{BaFe}_{9.6} \mathrm{Co}_{0.8} \mathrm{Ti}_{0.8} \mathrm{O}_{19}$ particles", Mater. Chem. Phys. 64 (2000) 256-259.

[6] M. S. Pinho, M. L.Gregori, R. C. R. Nunes, B. G. Soares, "Performance of radar absorbing materials by waveguide measurements for X and Ku-band frequencies", Eur. Polymer J. 38, 11 (2002) 2321-2327.

[7] S. C. Gupta, N. L. Agrawa, "Broad band thin sheet absorbers for S-; C-; X- and Ku- bands", J. IETE 39 (1993) 197-200.

[8] S. A. Papoullas, H. S. Wayne, "Radar absorber”, U S. Patent 5,147,718 (1992).

[9] I. Nedkov, A. Petkov, V. Karpov, "Microwave absorption in Sc- and CoTi- substituted Ba hexaferrite powders", Trans. Magnetics 26, 5 (1990) 1483 -1484.

[10] R. C. Lima, "Efeito da substituição de bário por lântâniosódio nas propriedades absorvedoras de microondas da hexaferrita de bário do tipo M", Dissertação de Mestrado, COPPE-UFRJ, Rio de Janeiro, RJ (2002) 100 p.

[11] J. L. Forveille, L. Olmedo, J. Raby, "Organic materials filled with ferrite powder for electromagnetic compatibility", J. Physique IV 7 (1997) C1-427/8.

[12] M. Pardavi-Horvath, "Microwave applications of soft ferrites", J. Magn. Magn. Mater. 215 (2000) 171-183.

[13] J. Baker-Jarvis, M. D. Janezic, J. H. Grosvenor, R. G. Geyer, "Theory for coaxial line and rectangular waveguide measurements of permittivity and permeability", NIST Technical Note 1355-R (1993) 40-51.

[14] American Society for Testing and Materials, vol. 02.05, Philadelphia, 1990 - Método ASTM B 117-90, "Standard Test Method of Salt Spray (Fog) Testing".

[15] M. S. Pinho, R. C. Lima, T. Ogasawara, F. T. Silva, "Influência nas medidas de refletividade do efeito de dispersão de partículas ferrimagnéticas em matrizes poliméricas", Pesquisa Naval 16 (2003) 73-80.

(Rec. 19/08/03, Rev. 05/11/03, Ac.12/04/04) 